\title{
The Role of Phytochrome in Photoperiodic Timing Mechanisms
}

\author{
James D. Metzger and Z. - L. Zheng \\ Department of Horticulture and Crop Science, The Ohio State University, 2021 Coffey Road, Columbus, OH 43210 U. S. A.
}

\begin{abstract}
Summary
For over 50 years it has been known that the chromoprotein phytochrome has a fundamental role in photoperiodic timing mechanisms. However, exactly how phytochrome is involved in measuring the length of the dark period has eluded all attempts by researchers to elucidate biochemical and molecular mechanisms. This predicament can be partly explained by the recent discovery that phytochrome is actually a family of a number of related photoreceptors with separate, but overlapping functions. We have been engaged in an attempt to delineate the roles of individual phytochromes in photoperiodic timing in both a LDP and a SDP through reverse genetics. We are using transgenic plants of Nicotiana sylvestris (a LDP) and Nicotiana tabacum cv. Maryland Mammoth (a SDP) that have been engineered to overproduce or underproduce either Phy A or Phy B using a double $35 \mathrm{~S}$ promoter to enhance expression of the transgenes. To date three homozygous antisense tobacco PHYA lines of $N$. sylvestris have been obtained; PCR analysis showed the integration of the transgene, and these lines displayed longer hypocotyls under continuous far red light. A total of five $N$. sylvestris lines expressing PHYB in the sense orientation have also been identified by PCR analysis. Seedlings of four of these putative $P H Y B$ overexpressing lines had significantly shorter hypocotyl lengths under continuous red light. Interestingly, one overexpressing $P H Y B$ line exhibited a phenotype consistent with co-suppression: under red light, hypocotyls of this line were much longer than those of wild type seedlings. We are now in the process of examining the photoperiodic responses in both classes of transgnic plants. We are also in the process of characterizing plants of $N$. sylvestris transformed with $P H Y B$ in the antisense orientation, as well as $N$. tobacum cv. Maryland Mammoth plants transformed with $P H Y B$ in both the sense and antisense orientation, and with PHYA in the antisense orientation. Further progress on the molecular characterization of these additional transgenic lines is reported.
\end{abstract}

\section{Introduction}

Living organisms, particularly those living in temperate climates, must be able to adjust to seasonal changes in temperature and water availability. These adjustments include hibernation and migratory behaviors in animals, development of resting spores in fungi, and various modifications in plant growth and development. In most cases these responses reflect adaptations to survive the stresses imposed by the cold temperatures of winter. Moreover, the predominant environmental cue that living organisms use as a signal for impending changes in season is the length of day or photoperiod.

Reproduction in both plants and animals must also be coordinated with the season since the reproductive cycle must be completed before the onset of winter or the dry season. In many plants the initiation of reproductive development or flowering is controlled by daylength. Photoperiodic control flowering was first demonstrated by Garner and Allard (1920) nearly 80 years ago. In the

This paper was presented at Session of Physiology and Biochemistry of Flowering, the Commemorative Symposium of the 75th Anniversary of Japanese Society for Horticultural Science held on 3rd and 4th of April, 1998. intervening years considerable work has been expended trying to elucidate photoperiodic mechanisms. While the central role of phytochrome in sensing light/dark transitions was recognized over 45 years ago (Borthwick et al., 1952), its function(s) in photoperiodic timing mechanisms remain obscure. The recent identification that phytochrome actually represents a family of chromoproteins with distinct, but overlapping functions (Smith, 1995), is a contributing factor in the difficulty in drawing definitive conclusions on its role in photoperiodic timing mechanism. Furthermore, we now know that plants measure the length of the dark period rather than the duration of the light period (Hamner, 1940; Hamner and Bonner, 1938), but it is unclear what are the underlying biochemical or molecular mechanisms for time measurement. Present work in my laboratory is directed towards determining how plants measure time. More specifically we are asking three questions: 1 ) what is the role of phytochrome; 2 ) is phytochrome an integral part of the timing mechanism; 3) which phytochrome gene(s) are involved. 


\section{Long - Day and Short - Day Response Systems: Identical Signal Transduction Pathways?}

It is often assumed that the photoperiodic response systems contained in long day plants (LDP) and short day plants (SDP) are mechanistically mirror images of each other. However, there is both genetic and physiological evidence that argues against this. I will first discuss the genetic evidence. Tobacco (Nicotiana tabacum) is a tetraploid hybrid of two diploid species: $N$. sylvestris, a LDP and $N$. tomentosiformis, a SDP. Most tobacco cultivars are day neutral plants (DNP) suggesting that the long day and short day response systems of the two parents are operating independently such that tobacco will flower at any daylength. However, the tobacco cultivar Maryland Mammoth is a SDP as a result of a single gene recessive mutation in the long day response mechanism originating from the $N$. sylvestris parent (Lang and Melchers, 1948).

There is also physiological evidence that the long day and short day response systems represent two separate signal transduction pathways. American willowherb (Epilobium ciliatum) is a perennial weed of orchards and container crops. This species overwinters by producing frost tolerant adventitious buds at the crown during late summer and early fall. However E. ciliatum flowers in early summer. Work in my laboratory has shown that flowering is a long day response with a critical daylength of 14.5 hours while adventitious bud formation is a short day response with a critical day length of about 13 hours (Przepiorkowski and Metzger, 1994). Thus $E$. ciliatum contains at least two mechanisms to measure time: one for long day responses and another for short day responses.

\section{Is Phytochrome an Integral Part of Photoperiodic Mechanisms?}

Although it is well known that phytochrome has an obligatory function in photoperiodic timing mechanisms, its specific role is still not known. Action spectra performed at different times of a day-night cycle showed that there is a diurnal cycle in the sensitivity to exposure to red and far-red light. In SDP, red light tends to promote flowering if presented during the light period of an inductive short day, while far-red is inhibitory if given at the same time. However, red light becomes increasingly inhibitory if given during an inductive night; this inhibition of flowering by red light reaches a maximum approximately half way through the dark period (for a review, see Bernier et al., 1981; Zeevaart, 1976). In general the sequence of sensitivity to red and far red light is reversed in long day plants in that far red light promotes flowering if given during the first half of an inductive dark period, while red light is promotive if given during the second half of an induc-

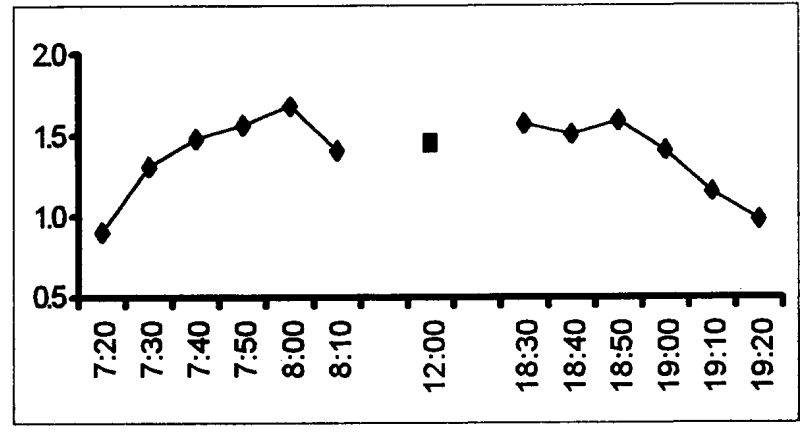

Fig. 1. Diurnal changes in the red / far-red ratio in Columbus, $\mathrm{OH}\left(40^{\circ} \mathrm{N}\right)$ on October 17.

tive dark period (Bernier et al., 1981; Zeevaart, 1976). There is also a substantial body of evidence that these diurnal changes in sensitivity to light quality are under the control of a circadian oscillator (reviewed in Bernier et al., 1981; Zeevaart, 1976; Lumsden, 1991).

Many of the earlier models invoke changes in the rate of the so called "dark reversion" of the red absorbing form of phytochrome ( $\mathrm{Pr})$ to the far-red absorbing form (Pfr) as a critical component in the photoperiodic timing mechanism (Bernier et al., 1981; Zeevaart, 1976). However, an equally plausible function of phytochrome may be related to measuring diurnal changes in the ratio of red to far-red light. As the end of the day approaches, the path length of sun light through the atmosphere increases resulting in a larger increase of scattering of red light over that of far-red light. As a consequence the ratio of red to far-red light gradually decreases until about 20 minutes after sunset, while the inverse sequence occurs at sunrise. Figure 1 shows the diurnal change in change in the red / far-red ratio on October 15 in Columbus, $\mathrm{OH}\left(40{ }^{\circ} \mathrm{N}\right)$. This increase in the proportion of far-red light that occurs daily is responsible for the induction of end of the day (EOD) responses. An example of an EOD response is the increase in stem growth that occurs in many species when plants are illuminated for short periods ( 1 hour or less) just before total darkness with light of a red/far-red ratio of 1 or less (Kasperbauer, 1971; Kasperbauser et al., 1984). The increase in the proportion of far red light as dusk approaches is probably an important cue for signaling the impending night. Thus the EOD far-red signal may initiate the photoperiodic timing mechanism analogous to the start button on a stopwatch. This is consistent with the hourglass mechanism for photoperiodic time measurement proposed by Borthwick and Hendricks (1960).

However, this model can not explain photoperiodic timing mechanism in many species especially LDP (Bernier et al., 1981). As mentioned above there is a diurnal cyclic alteration in sensitivity to light such that far-red and red light may alternately inhibit or promote flowering with the specific response dependent on the time of day and the response type of the species. It may be that the processes associated with flower induction are promoted when the EOD far-red light signal coin- 
cides with the time of the day when far-red light is promotive or has a neutral effect on flowering. The EOD far-red signal is not the only light cue involved either, since red light can be promotive or inhibitory depending on the time of day.

\section{Molecular Genetics, Arabidopsis, and the Analysis of Photoperiodic Mechanisms}

The molecular and biochemical basis of photoperiodic timing mechanisms remains virtually unknown. Part of reason for this is that the best systems for physiological studies do not lend themselves very well to genetic and/or molecular analysis. While on the other hand, the use of Arabidopsis thaliana, the model system of choice for molecular biologists, has several limitations for studies on photoperiodism. The chief problem is that although Arabidopsis is considered a quantitative LDP, certain ecotypes do not display a LD response similar to other LDP. This is acutely apparent when the early flowering Landsberg erecta ecotype is the subject of physiological studies. Unlike most other LDP, LDinduced promotion of flowering is only achieved when day length extensions contain far-red light (Bagnall, 1992; Bagnall et al., 1995).

In order to exploit the potential of Arabidopsis as system to dissect the molecular mechanisms of underlying photoperiodic timing, it is necessary to select the appropriate genotype that exhibits the proper physiological responses to LD. We began our search by screening plants of the ecotype Columbia (CS933, ABRC) for delayed flowering in SD. A line was selected in which the delay of flowering in SD was eliminated by extending a 9 -hr SD photoperiod consisting of light from fluorescent and incandescent lamps $\left(400 \mu \mathrm{mol} \mathrm{m}^{-2}\right.$ - $\mathrm{s}^{-1}$ PAR) with $6 \mathrm{hr}$ of low intensity light from incandescent lamps (30 $\mu \mathrm{mol} \mathrm{m}^{-2} \mathrm{~s}^{-1}$ PAR). The red/far-red ratio $(650-670 \mathrm{~nm} / 720-740 \mathrm{~nm})$ was 1.6 and 1.05 for the main photoperiod and the daylength extension, respectively. As can be seen in Fig. 2, flowering times were reduced with increasing number of LD. Never-

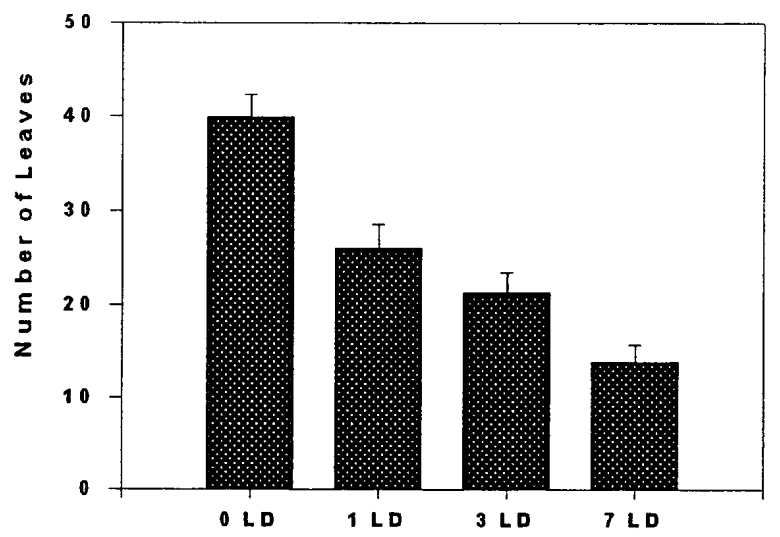

Fig. 2. Effect of various numbers of long days on flowering times in CS933.

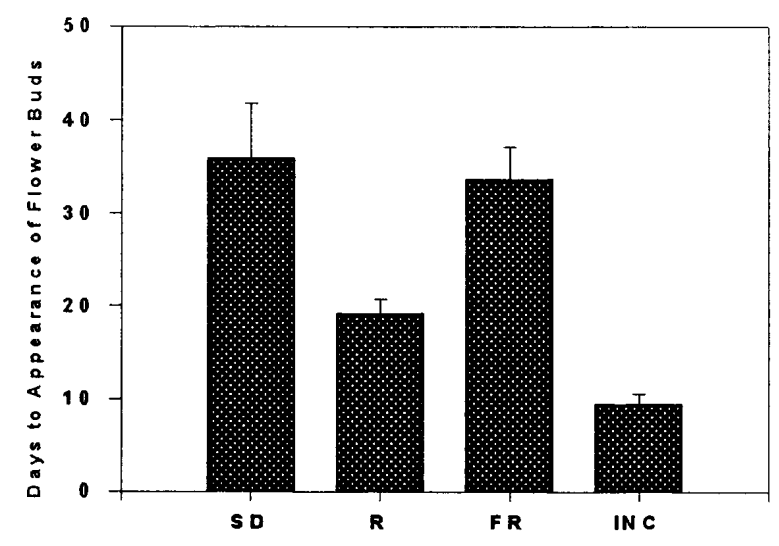

Fig. 3. Effect of light quality of day length extensions on flowering times in CS933.

theless, flowering was significantly promoted even when plants were exposed to only $1 \mathrm{LD}$ and then returned to SD.

Light quality of the daylength extension greatly affected the LD response of this line. Short $(9 \mathrm{hr})$ photoperiods were extended with $6 \mathrm{hr}$ of low intensity red (660 nm peak), far red ( $730 \mathrm{~nm}$ peak), or light from incandescent lamps. Flowering was promoted only when the day length extension contained red light, although maximum promotion of flowering was obtained with a mixture of red and far-red light from incandescent lamps (Fig. 3). This result is in contrast with workers using the early flowering ecotype Landsberg, who found that only daylength extensions with far-red light were effective in promoting flowering (Bagnall, 1992; Bagnall et al., 1995). However, most other LDP respond to daylength extensions of different light qualities as shown in Fig. 3 for CS933 (Lumsden, 1991 and references cited within).

Although daylength extensions consisting of far-red

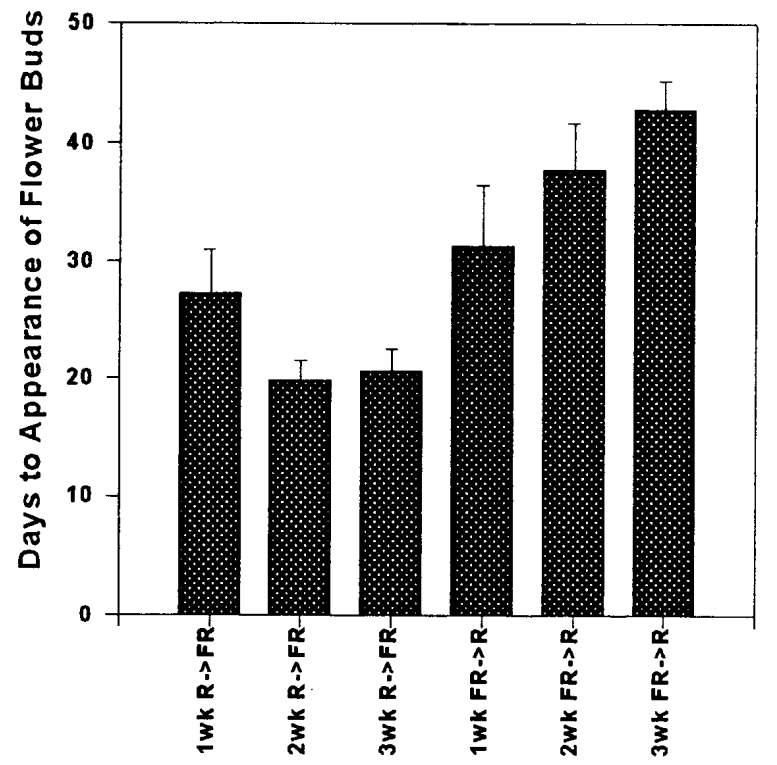

Fig. 4. Effect of sequence of light quality of day length extensions on flowering times in CS933. 
light alone did not induce flowering, its addition to red light on an approximately 1:1 basis from incandescent lamps increased the inductive effect of the red daylength extension (Fig. 3). This raises the question whether farred light directly interacts with the photoperiodic timing mechanism or has some indirect effect on flowering. This was tested by sequentially extending the SD with either red or far-red light for 1, 2, or 3 weeks, followed by daylength extensions using the other light quality. As can be seen in Fig. 4, the greatest promotion of flowering was observed when plants received red daylength extensions for two or more weeks. When the far-red extensions were provided first, no promotion of flowering was seen until the plants were subjected to the red daylength extensions.

One interpretation of these results is that red and farred light affect a different aspect of flowering. It is clear that red light is required for flowering initiation since flowering occurs only when the daylength extension contains this light quality (Figs. 3 and 4). On the other hand, when far-red light is added to the daylength extension, further development of the flower primordium and inflorescence may be promoted. Thus, far-red light affects flowering only after the flower initiation has occurred. Downs and Thomas (1982) made the same conclusion 16 years ago for the independent actions of red and far-red light on flowering for the LDP Hyoscyamus niger.

During the course of this work, we have also discovered that the method by which we measured flowering strongly determines the conclusions that are drawn from that data. When flowering was assessed by days to the appearance of flower buds (Fig.3), extending SD with far-red light alone clearly was not sufficient to promote flowering. However, if leaf number is used as the measure of flowering, far-red extensions do indeed significantly promote flowering (Fig. 5). However, inasmuch as far-red light reduce leaf initiation (Smith, 1975), leaf number is therefore not a stable develop-

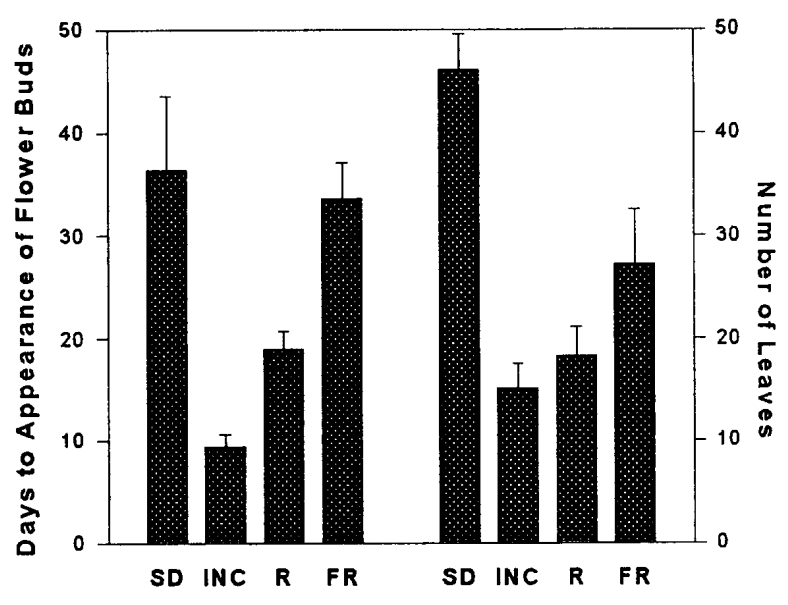

Fig. 5. Effect of light quality of day length extensions on flowering times in CS933 as measured by two different methods. mental parameter against which one can normalize other developmental phenomena.

\section{Which Phytochrome Genes Are Involved in Photoperiodic Timing?}

Crucial to determining the role of phytochrome in photoperiodic timing mechanisms is delineating the functions of the individual phytochromes. Physiological analysis of plants with loss of function mutations in the various phytochrome genes has provided some clues about their possible functions. Mutations resulting in the impaired function of phy $\mathrm{B}$ have been identified in the LDP pea (Weller et al., 1985) and Arabidopsis (Reed et al., 1994) as well as the SDP sorghum (Childs et al., 1997). In all three cases, mutation of the phy B gene resulted in plants that flower early in non-inductive conditions. In addition, transgenic potato plants expressing an antisense phy B genes form tubers (a short day process) in long day (non-inductive) conditions (Jackson et al., 1996). This has led to the suggestion that phy $\mathrm{B}$ controls flowering by modulating the production of a graft-transmissible flowering inhibitor (Jackson and Thomas, 1997).

Mutations in the phy A gene in Arabidopsis (Reed et al., 1994) and pea (Weller et al., 1997) also alter LD photoperiodic timing mechanisms. Loss of phy A function in these two species results in the delay of flowering under inductive conditions. Thus, in contrast to the inhibitory role of phy $B$, phy $A$ serves as a positive regulator of flowering. It was suggested that phy A also controls flowering through control of the either the synthesis or the transport of a flowering inhibitor (Weller et al., 1997).

Although the idea that phy A and phy B interact in the photoperiodic control of flowering through modulating the level or transport of an inhibitor is appealing, it may be too simplistic. The fact that red light can alternatively promote or inhibit flowering in SDP depending on the time of the day is not easily explained by phytochrome control of inhibitor levels.

We have begun investigating the roles of phy $\mathrm{A}$ and phy $\mathrm{B}$ in photoperiodic timing mechanisms using an approach different than mutational analysis. We are utilizing reverse genetics and exploiting the existence of both long and short day response types in Nicotiana species. We are attempting to generate transgenic plants of Nicotiana sylvestris (a LDP) and Nicotiana tabacum cv. Maryland Mammoth (a SDP) that overexpress or underexpress (antisense) either phy A or phy B.

The effect of over- or underproduction of the two phytochrome genes on the photoperiodic responses will be examined. To date we have been successful in generating phy $\mathrm{A}$ antisense and phy $\mathrm{B}$ overexpressing plants of $N$. sylvestris. Southern and reverse-PCR analysis of these plants showed that the transgenes were incorporated into the genome and were being expressed 


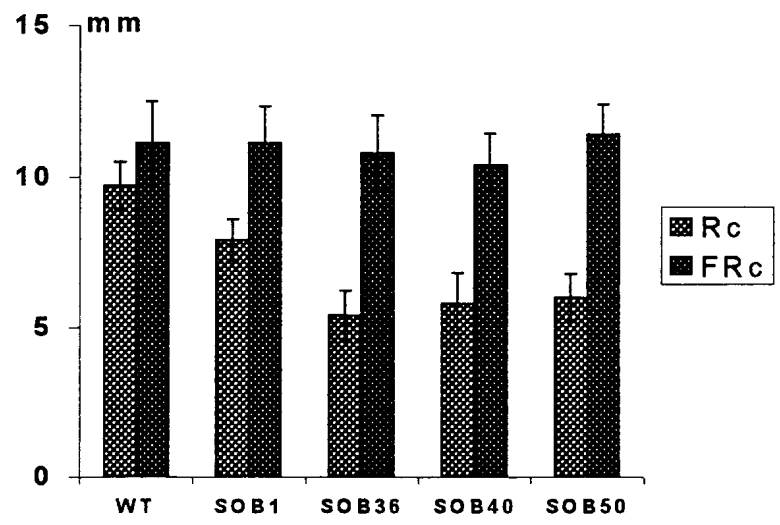

Fig. 6. Hypocotyl growth of putative phy B overexpressing lines of $\mathrm{N}$. sylvestris under continuous red (Rc)or far-red (FRc) light.

(data not shown). Physiological analysis of hypocotyl growth of the transgenic plants under continuous red and far-red light showed that the plants responded in a manner predicted by their respective genotypes.

Phy B over expression in Arabidopsis seedlings results in increased inhibition of hypocotyl growth by red light; the response to far-red light is unaffected (Reed et al., 1994). Several transgenic lines of $N$. sylvestris overexpressing phy $\mathrm{B}$ exhibit a similar response to red and far-red light (Fig. 6). In contrast, phy A loss of function mutations in Arabidopsis result in a loss of the inhibitory response in hypocotyl growth to far-red light (Reed et al., 1994). A similar response was observed in several anti-sense phy A $N$. sylvestris lines (Fig. 7).

We are currently in the process of examining the photoperiodic behavior of these plants. Transgenic plants have also been generated in which, as indicated by Southern and reverse-PCR analysis, we have lines of $N$. sylvestris and $N$. tabacum cv. Maryland Mammoth that under-express phy $\mathrm{B}$ and anti-sense phy A lines of $N$. tabacum. The physiological characterization of hypocotyl growth is currently under way to ensure the proper expression of the transgenes is occurring.

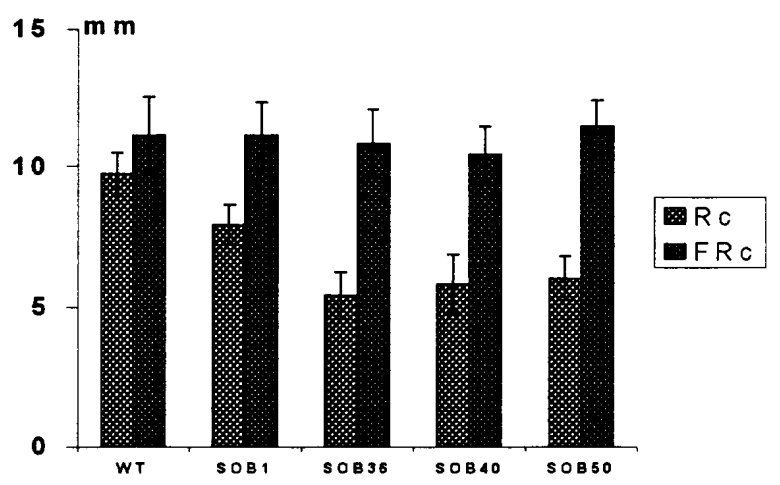

Fig. 7. Hypocotyl growth of putative phy A underexpressing lines of $N$. sylvestris under continuous red $(\mathrm{Rc})$ or farred (FRc) light.

\section{Literature Cited}

Bagnall, D. J. 1992. Control of flowering in Arabidopsis thaliana by light, vernalization and gibberellins. Aust. J. Plant Physiol. 41 : 401-409.

Bagnall, D. J., R. W. King, G. C. Whitelam, M. T. Boylan, D. Wagner and P. H. Quail. 1995. Flowering responses to altered expression of phytochrome in mutants and transgenic lines of Arabidopsis thaliana (L.) Heynh. Plant Physiol. 108 : 1495-1503.

Bernier, G., J-M. Kinet and R. M. Sachs. 1981. The Physiology of Flowering,. Vol. I. CRC Press, Boca Raton.

Borthwick, H. A. and S. B. Hendricks. 1960. Photoperiodism in plants. Science $132: 1223-1228$.

Borthwick, H. A., S. B. Hendricks and M.W. Parker. 1952. The reaction controlling flower initiation. Proc. Natl. Acad. Sci. USA. $38: 929-934$.

Childs, K. L., F. R. Miller, M-M. Cordonnier-Pratt, L. H. Pratt, P. W. Morgan and J.E. Mullet. 1997. The sorghum photoperiod sensitivity gene, Ma3, encodes a phytochrome B. Plant Physiol. 113 : 611-619.

Downs, R. J. and J. F. Thomas. 1982. Phytochrome regulation of flowering in the long-day plant Hyoscyamus niger. Plant Physiol. 70:898-900.

Garner, W. W. and H. A. Allard. 1920. Effect of the relative length of day and night and other factors of the environment on growth and reproduction in plants. J. Agric. Res. $18: 553-606$.

Hamner, K. C. 1940. Interrelation of light and darkness in photoperiodic induction. Bot. Gaz. 101 : 658-687.

Hamner, K. C. and J. Bonner. 1938. Photoperiodism in relation to hormones as factors in floral initiation and development. Bot. Gaz. 100 : 388-431.

Jackson, S. D., A. Heyer, J. Dietze and S. Prat. 1996. Phytochrome B mediates the photoperiodic control of tuber formation in potato. Plant Journal $9: 159-166$.

Jackson, S. and B. Thomas. 1997. Photoreceptors and signals in the photoperiodic control of development. Plant Cell and Env. $20: 790-795$.

Kasperbauer, M. J . 1971. Spectral distribution of light in a tobacco canopy and effects of light quality on growth and development. Plant Physiol. 47 : 775-778.

Kasperbauer, M. J., P. G. Hunt and R. E. Sojika. 1984. Photosynthate partitioning and nodule formation in soybean plants that received red or far-red light at the end of the photosynthetic period. Plant Physiol. 61 : 549 -554 .

Lang, A. and G. Melchers. 1948. Auslösung der Blütenbildung bei Langtagpflanzen unter Kurztagbedingungen durch Aufpfropfung von Kurztagpflanzen. Zeitschrift Naturforsch. 3 : 108-111.

Lumsden, P. J. 1991. Circadian rhythms and phytochrome. Annu. Rev. Plant Physiol. Plant Mol. Biol. 42 : 351 371.

Przepiorkowski, T. M. and J. D. Metzger. 1996. Environmental control of perennating structure formation in American willowherb (Epilobium ciliatum). Weed Science $37: 116$ (Abstract) 
Reed, J. W., A. Nagatani, T. D. Elich, M. Fagan and J. Chory. 1994. Phytochrome A and phytochrome B have overlapping but distinct functions in Arabidopsis development. Plant Physiol. 104 : 1139 - 1149.

Smith, H. 1975. Phytochrome and photomorphogenesis : An introduction to the photocontrol of plant development. McGraw - Hill, London.

Smith, H. 1995. Physiological and ecological function within the phytochrome family. Annu. Rev. Plant Physiol. Plant Mol. Biol. 46 : 289-316.
Weller, J. L., I. C. Murfet and J. B. Reid.1997. Pea mutants with reduced sensitivity to far-red light define an important role for phytochrome $\mathrm{A}$ in day-length detection. Plant Physiol. 114 :1225-1236.

Weller, J. L., A. Nagatani, R. E. Kendrick, I. C. Murfet and J. B. Reid. 1995. New $l v$ mutants of pea are deficient $I$ phytochrome B. Plant Physiol. 108 : 525- 532.

Zeevaart, J. A. D. 1976. Physiology of flower formation. Annu. Rev. Plant Physiol. 27 : 321-348. 